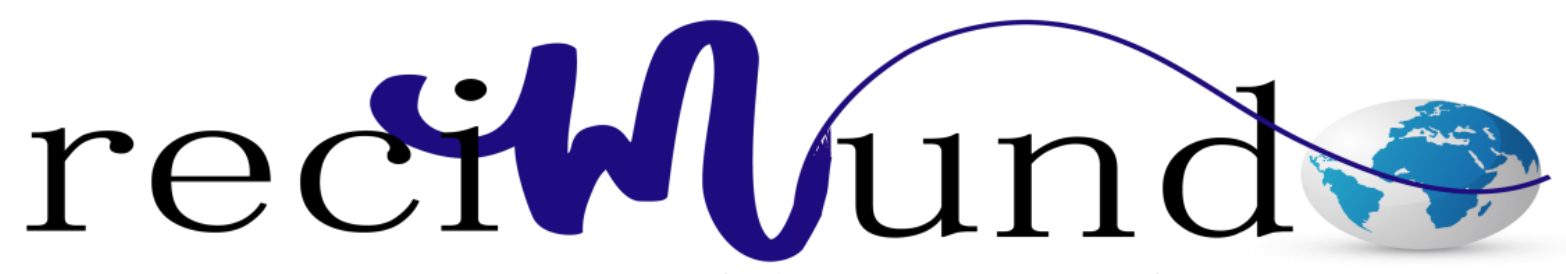

Revista Científica Mundo de la Investígación y el Conocimiento

\begin{abstract}
Jaqueline Magaly Quisanga Llumiluisa a; Nery Hermelinda Balseca Guaman ${ }^{\text {b; }}$
Marco Antonio Ipiales Miranda ${ }^{c}$; Cristina Mishell Zurita Pozo ${ }^{d}$
\end{abstract}

Atención y cuidado en el paciente con tuberculosis por derrame pleural

Care in patients with tuberculosis due to pleural effusion

Revista Científica Mundo de la Investigación y el Conocimiento. Vol. 3 núm.3, septiembre, ISSN: 2588-073X, 2019, pp. 1270-1293

DOI: $\underline{10.26820 / \text { recimundo/3.(3).septiembre.2019.1270-1293 }}$

URL: http://recimundo.com/index.php/es/article/view/569

Código UNESCO: 3205 Medicina Interna

Tipo de Investigación: Artículo de Revisión

Editorial Saberes del Conocimiento

Recibido: 15/05/2019

Aceptado: 23/06/2019

Publicado: 30/09/2019

Correspondencia: jaquelinq_33@hotmail.com

a. Médico; Investigador Independiente; Guayaquil, Ecuador; jaquelinq_33@ hotmail.com

b. Médico; Investigador Independiente; Guayaquil, Ecuador; nery_balseca@ hotmail.com

c. Médico; Investigador Independiente; Guayaquil, Ecuador; marko88riot@ gmail.com

d. Médico; Investigador Independiente; Guayaquil, Ecuador; cristinapichell@ hotmail.com 


\section{Atención y cuidado en el paciente con tuberculosis por derrame pleural}

Vol. 3, núm. 3., (2019)

Jaqueline Magaly Quisanga Llumiluisa; Nery Hermelinda Balseca Guaman; Marco Antonio Ipiales Miranda; Cristina Mishell Zurita Pozo

\section{RESUMEN}

El proceso evolutivo del conocimiento ha venido de la mano del desarrollo de investigaciones y estas a su vez han servido de herramientas para el desarrollo de los diferentes enfoques pedagógicos de la educación. La utilización de herramientas pedagógicas para transferir el conocimiento ha sido de vital importancia ya que allí se genera el conocimiento sobre temas en específico. Estas herramientas han evolucionado a través del tiempo y han permitido acercar más a los individuos al conocimiento que desean adquirir quebrando viejos paradigmas incrustados en la sociedad. Es aquí donde entran las TIC como herramientas de desarrollo del conocimiento. Las redes sociales están jugando un papel importante en la masificación de conocimiento en este mundo globalizado. Por este motivo, esta investigación tiene como objetivo general analizar el uso de las redes sociales para actividades académicas colaborativas en la educación superior. La metodología empleada se basó en la utilización de investigación documental y bibliográfica. Los resultados que se obtuvieron se basaron en las características de las TIC, la realidad de la universidad ecuatoriana con las TIC y las redes sociales y las universidades ecuatorianas. Como conclusión se obtuvo que el uso de las redes sociales permite la masificación del conocimiento, a través de las distintas plataformas virtuales, haciendo que los nuevos profesionales tengan mayor pertenencia en su labor y aunado a esto un mayor compromiso social ya que fomentan el autoaprendizaje, desarrollan habilidades, fomentan el pensamiento crítico y comprenden la existencia de la diversidad.

Palabras Claves: Investigación; conocimiento; TIC; redes sociales. 


\title{
Atención y cuidado en el paciente con tuberculosis por derrame pleural
}

Vol. 3, núm. 3., (2019)

Jaqueline Magaly Quisanga Llumiluisa; Nery Hermelinda Balseca Guaman; Marco Antonio Ipiales Miranda; Cristina Mishell Zurita Pozo

\begin{abstract}
The evolutionary process of knowledge has come hand in hand with the development of research and these in turn have served as tools for the development of different pedagogical approaches to education. The use of pedagogical tools to transfer knowledge has been of vital importance since there is generated knowledge on specific topics. These tools have evolved over time and have allowed individuals to get closer to the knowledge they wish to acquire by breaking old paradigms embedded in society. This is where ICTs come in as tools for knowledge development. Social networks are playing an important role in the massification of knowledge in this globalized world. For this reason, this research has as its general objective to analyze the use of social networks for collaborative academic activities in higher education. The methodology used was based on the use of documentary and bibliographic research. The results obtained were based on the characteristics of ICT, the reality of the Ecuadorian university with ICT and social networks and Ecuadorian universities. As a conclusion it was obtained that the use of social networks allows the massification of knowledge, through the different virtual platforms, making new professionals have greater belonging in their work and coupled with this a greater social commitment as they promote self-learning, they develop skills, foster critical thinking and understand the existence of diversity.
\end{abstract}

Key Words: Research; knowledge; ICT; social networks. 


\section{Atención y cuidado en el paciente con tuberculosis por derrame pleural}

Vol. 3, núm. 3., (2019)

Jaqueline Magaly Quisanga Llumiluisa; Nery Hermelinda Balseca Guaman; Marco Antonio Ipiales Miranda; Cristina Mishell Zurita Pozo

\section{Introducción.}

La tuberculosis (TB) es una de las enfermedades infecciosas con mayor morbimortalidad a nivel mundial, por lo que constituye un auténtico problema de salud pública. La Organización Mun-dial de la Salud estima que durante el año 2011 se produjeron 8,7 millones de nuevos casos de TB, lo que equivale a una incidencia global de 125/100.000 habitantes/año y que murieron 1,4 millones de personas. La confección de la TB con el virus de la inmunodeficiencia humana (VIH), junto con la inmigración, han hecho que la TB resurgiera en los países desarrollados y que aumente la carga de la enfermedad en los que están en desarrollo (World Health Organization, 2012). El derrame pleural tuberculoso (DPTB) constituye un problema clínico frecuente en muchos países y es particularmente relevante en la etapa actual de infección por VIH, situación en la que es más frecuente en la práctica clínica.

El diagnóstico diferencial de derrames pleurales con exudado con predominio de linfocitos requierecuidadosa exploración. Los parámetros que generalmente están disponibles y comúnmente se miden en pleurallos derrames son útiles para la separación de trasudados de exudados, pero a menudo no contribuyen al diagnóstico de la enfermedad subyacente. Además, los procedimientos que conducen a un diagnóstico definitivo(es decir, biopsia y / o cultivo) podría ocasionar un retraso que amenace el manejo adecuado de la enfermedad y su desenlace. En la práctica diaria, generalmente es difícil diferenciar tuberculoso (TBE) de derrame maligno (DM) en ausencia de otros signos o manifestaciones radiológicas de la enfermedad. TBE puede ocurrir en ausencia de otra evidencia radiológica de tuberculosis pulmonar y puede ser debido a la enfermedad primaria o extra primaria, según la población estudiada. La pleuresía tuberculosa 


\section{Atención y cuidado en el paciente con tuberculosis por derrame pleural}

Vol. 3, núm. 3., (2019)

Jaqueline Magaly Quisanga Llumiluisa; Nery Hermelinda Balseca Guaman; Marco Antonio Ipiales Miranda; Cristina Mishell Zurita Pozo

es la forma más común de tuberculosis extrapulmonar. La presencia de células malignas en derrame pleural o tejido pleural se observa en diseminado o enfermedad neoplásica avanzada y conduce a malos resultados, destacando su importancia como factor de pronóstico. Actualmente, el cáncer de pulmón y de mama son los tumores metastásicos más comunes de la pleura en hombres y mujeres, respectivamente. En conjunto, ambas neoplasias representan aproximadamente el $70 \%$ de todos los tumores malignos y derrames pleurales (Valdés, Álvarez, Valle, Pose, \& San José, 1996).

\section{Metodología.}

Para el desarrollo de este proceso investigativo, se plantea como metodología la encaminada hacia una orientación científica particular que se encuentra determinada por la necesidad de indagar en forma precisa y coherente una situación, en tal sentido (Davila, 2015) define la metodología "como aquellos pasos previos que son seleccionados por el investigador para lograr resultados favorables que le ayuden a plantear nuevas ideas”. (p.66)

Lo citado por el autor, lleva a entender que el desarrollo de la acción investigativa busca simplemente coordinar acciones enmarcadas en una revisión bibliográfica con el fin de complementar ideas previas relacionadas Atención y cuidado en el paciente con tuberculosis por derrame pleurala través de una revisión de literatura, para así finalmente elaborar un cuerpo de consideraciones generales que ayuden a ampliar el interés propuesto. 


\section{Atención y cuidado en el paciente con tuberculosis por derrame pleural}

Vol. 3, núm. 3., (2019)

Jaqueline Magaly Quisanga Llumiluisa; Nery Hermelinda Balseca Guaman; Marco Antonio Ipiales Miranda; Cristina Mishell Zurita Pozo

Tipo de Investigación

Dentro de toda práctica investigativa, se precisan acciones de carácter metodológico mediante las cuales, se logra conocer y proyectar los eventos posibles que la determinan, así como las características que hacen del acto científico un proceso interactivo ajustado a una realidad posible de ser interpretada. En este sentido, se puede decir, que la presente investigación corresponde al tipo documental, definido por Castro (2016), "se ocupa del estudio de problemas planteados a nivel teórico, la información requerida para abordarlos se encuentra básicamente en materiales impresos, audiovisuales y /o electrónicos”. (p.41).

En consideración a esta definición, la orientación metodológica permitió la oportunidad de cumplir con una serie de actividades inherentes a la revisión y lectura de diversos documentos donde se encontraron ideas explicitas relacionadas con los tópicos encargados de identificar a cada característica insertada en el estudio. Por lo tanto, se realizaron continuas interpretaciones con el claro propósito de revisar aquellas apreciaciones o investigaciones propuestas por diferentes investigadores relacionadas con el tema de interés, para luego dar la respectiva argumentación a los planteamientos, en función a las necesidades encontradas en la indagación.

\section{Fuentes Documentales}

El análisis correspondiente a las características que predomina en el tema seleccionado, llevan a incluir diferentes fuentes documentales encargadas de darle el respectivo apoyo y en ese sentido cumplir con la valoración de los hechos a fin de generar nuevos criterios que sirven de referencia a otros procesos investigativos. Para (CASTRO, 2016) las fuentes documentales 


\section{Atención y cuidado en el paciente con tuberculosis por derrame pleural}

Vol. 3, núm. 3., (2019)

Jaqueline Magaly Quisanga Llumiluisa; Nery Hermelinda Balseca Guaman; Marco Antonio Ipiales Miranda; Cristina Mishell Zurita Pozo

incorporadas en la investigación documental o bibliográfica, "representa la suma de materiales sistemáticos que son revisados en forma rigurosa y profunda para llegar a un análisis del fenómeno". (p.41). Por lo tanto, se procedió a cumplir con la realización de una lectura previa determinada para encontrar aquellos aspectos estrechamente vinculados con el tema, con el fin de explicar mediante un desarrollo las respectivas apreciaciones generales de importancia.

\section{Técnicas para la Recolección de la Información}

La conducción de la investigación para ser realizada en función a las particularidades que determinan a los estudios documentales, tiene como fin el desarrollo de un conjunto de acciones encargadas de llevar a la selección de técnicas estrechamente vinculadas con las características del estudio. En tal sentido, (Bolívar, 2015), refiere, que es "una técnica particular para aportar ayuda a los procedimientos de selección de las ideas primarias y secundarias”. (p. 71).

Por ello, se procedió a la utilización del subrayado, resúmenes, fichaje, como parte básica para la revisión y selección de los documentos que presentan el contenido teórico. Es decir, que mediante la aplicación de estas técnicas se pudo llegar a recoger informaciones en cuanto a la revisión bibliográfica de los diversos elementos encargados de orientar el proceso de investigación. Tal como lo expresa, (Bolívar, 2015) "las técnicas documentales proporcionan las herramientas esenciales y determinantes para responder a los objetivos formulados y llegar a resultados efectivos" (p. 58). Es decir, para responder con eficiencia a las necesidades investigativas, se introdujeron como técnica de recolección el método inductivo, que hizo posible llevar a cabo una valoración de los hechos de forma particular para llegar a la explicación desde una visión general. 


\section{Atención y cuidado en el paciente con tuberculosis por derrame pleural}

Vol. 3, núm. 3., (2019)

Jaqueline Magaly Quisanga Llumiluisa; Nery Hermelinda Balseca Guaman; Marco Antonio Ipiales Miranda; Cristina Mishell Zurita Pozo

Asimismo, se emplearon las técnicas de análisis de información para la realización de la investigación que fue ejecutada bajo la dinámica de aplicar diversos elementos encargados de determinar el camino a recorrer por el estudio, según, (Bolívar, 2015) las técnicas de procesamiento de datos en los estudios documentales "son las encargadas de ofrecer al investigador la visión o pasos que debe cumplir durante su ejercicio, cada una de ellas debe estar en correspondencia con el nivel a emplear" (p. 123). Esto indica, que para llevar a cabo el procesamiento de los datos obtenidos una vez aplicado las técnicas seleccionadas, tales como: fichas de resumen, textual, registros descriptivos entre otros, los mismos se deben ajustar al nivel que ha sido seleccionado.

\section{Resultados.}

\section{Epidemiología}

El DPTB es en nuestra región la causa más frecuente de TB extrapulmonar y la tendencia epidemiológica de los DPTB sigue una disminución significativa, ya que durante el periodo 2000-2009 tanto el número de casos como la incidencia se redujeron a la mitad (DetallePublicacion.aspx, 2009). Sin embargo, la proporción de pacientes con un DPTB sobre el total de casos de TB no ha cambiado de forma significativa (entre el 14,3 y el 19,3\%). Estos porcentajes son considerablemente mayores que los registrados en Estados Unidos $(3,6 \%)$ e inferiores a los de algunos países africanos (mayores del 20\%). Estas diferencias pueden atribuirse a las distintas prevalencias de TB en la poblacióngeneral o a que, al ser el cultivo del líquido pleural (LP) habitualmente negativo, la incidencia de DPTB puede subestimarse (Light, 2010). 


\section{Atención y cuidado en el paciente con tuberculosis por derrame pleural}

Vol. 3, núm. 3., (2019)

Jaqueline Magaly Quisanga Llumiluisa; Nery Hermelinda Balseca Guaman; Marco Antonio Ipiales Miranda; Cristina Mishell Zurita Pozo

El DPTB es más frecuente en hombres $(63,5 \%)$, en edades entre los $15-44$ años $(61,2 \%)$ y en los pacientes VIH positivos. Aunque sería esperable que fuera más frecuente en los pacientes inmunocompetentes que en los que presenten una alteración de la inmunidad celular, generalmente no es así (Awil, Bowlin, \& Daniel, 1997).

Figura 1. Esquema de las vías y biomarcadores involucrados en la respuesta inmunológica de los derrames pleurales tuberculosos

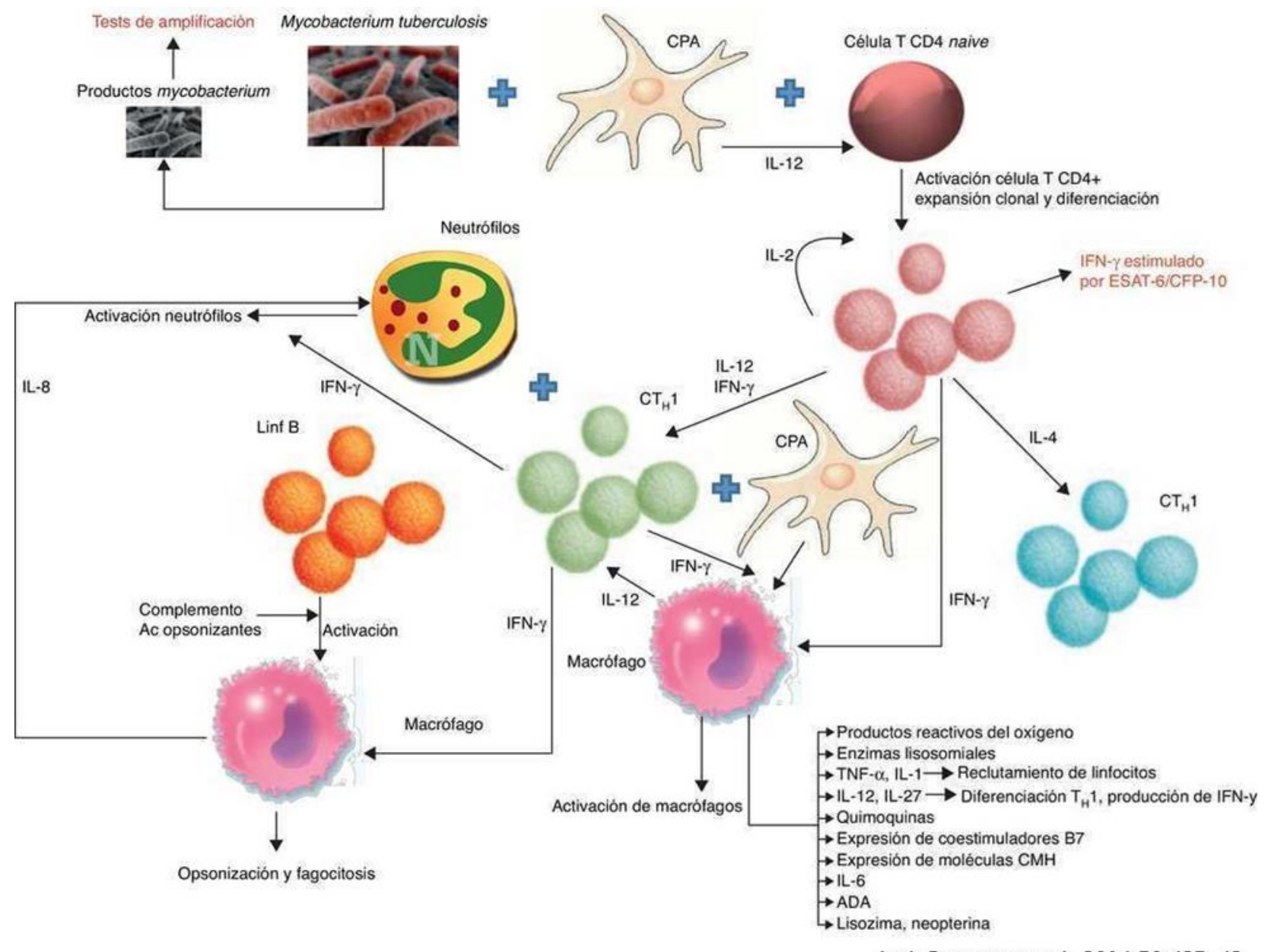

Arch Bronconeumol. 2014;50:435-43 


\section{Atención y cuidado en el paciente con tuberculosis por derrame pleural}

Vol. 3, núm. 3., (2019)

Jaqueline Magaly Quisanga Llumiluisa; Nery Hermelinda Balseca Guaman; Marco Antonio Ipiales Miranda; Cristina Mishell Zurita Pozo

\section{Patogenia}

El DPTB puede ser una manifestación tanto de una infección primaria como de una reactivación de la enfermedad. Esta última es la que predomina en los países desarrollados. Se cree que el DPTB se origina tras la rotura de un foco caseoso subpleural que libera su contenido en el espacio pleural. Los antígenos micobacterianos interaccionan con los linfocitos T-CD4+ dando lugar a una reacción de hipersensibilidad retardada en la que intervienen distintas citoquinas que tratan de estimular la acción antimicobacteriana de los macrófagos (fig. 1). Todo ello produce un aumento de la permeabilidad capilar y un deterioro del drenaje linfático, que origina el derrame pleural (DP). Esta teoría se apoya en que el cultivo del LP de estos pacientes suele ser negativo y que cuando se inyecta una proteína tuberculosa en el espacio pleural de cobayas previamente sensibilizados con derivados proteicos purificados se produce un DP exudativo que desaparece cuando a estos animales se les trata con suero anti linfocítico (Leibowitz, Kennedy, \& Lessof, 1973).

El empiema tuberculoso, una infección activa crónica del espacio pleural, puede producirse como consecuencia de la extensión dela infección desde otras localizaciones, tras una neumonectomía, o cuando una fístula broncopleural vierte el contenido de una cavidad en el espacio pleural. Un DPTB también puede originar un engrosamiento fibroso de la pleura visceral que impida la expansión del pulmón (pulmón atrapado). En estos casos se creará una presión negativa en el espacio pleural que favorecerá el desarrollo de un DP crónico, con características propias, aunque la enfermedad no esté activa (Pereyra, Ferreiro, \& Valdés, 2013). 


\section{Atención y cuidado en el paciente con tuberculosis por derrame pleural}

Vol. 3, núm. 3., (2019)

Jaqueline Magaly Quisanga Llumiluisa; Nery Hermelinda Balseca Guaman; Marco Antonio Ipiales Miranda; Cristina Mishell Zurita Pozo

\section{Clínica}

En países con alta incidencia de TB, la edad media de los pacientes con un DPTB oscila entre los 32 y los 34 años y el 70\% tienen menos de 40 años. En Estados Unidos la media de edad de presentación es de 49 años; solamente el 50\% son menores de 45 y el 30\% tienen más de 65 años (Baumann, y otros, 2007). Para justificar estas diferencias se ha sugerido que en los países desarrollados el DPTB podría ser el resultado de una reactivación, mientras que en los que aparece a edades más tempranas correspondería a una forma primaria de la enfermedad.

La sintomatología de los pacientes con un DPTB es variable. La fiebre $(86 \%$ de los casos), el dolor torácico de características pleuríticas (75\%) y la tos (70\%) suelen estar presentes28,29 y pueden acompañarse de otros síntomas de afectación sistémica. La clínica varía en función de la población. En los ancianos el periodo sintomático tiende a ser más prolongado, mientras que los pacientes VIH positivos son, normalmente, menos sintomáticos. Por el contrario, los jóvenes suelen presentar una clínica más aguda, con fiebre elevada.

El DPTB suele ser unilateral y de cualquier tamaño. En una serie de 254 casos, el 98,5\% eran unilaterales (56\% del lado derecho). Los DPTB masivos son poco habituales pero, no obstante, son la tercera causa más frecuente de los mismos (Porcel \& Vives, Etiology and pleural fluid characteristics of large and mas-sive effusions, 2003). La radiografía de tórax demuestra afectación pulmonar en el $25 \%$ de los casos, pero puede sobrepasar el $50 \%$ si se hace una tomografía computarizada (TC) de tórax. El porcentaje de afectación pulmonar dependerá de si el DPTB se produce como consecuencia de una infección pri-maria o de una reactivación (donde presumiblemente será más elevado). La tercera parte de los DPTB tendrá un Mantoux negativo. 


\section{Atención y cuidado en el paciente con tuberculosis por derrame pleural}

Vol. 3, núm. 3., (2019)

Jaqueline Magaly Quisanga Llumiluisa; Nery Hermelinda Balseca Guaman; Marco Antonio Ipiales Miranda; Cristina Mishell Zurita Pozo

En estos casos debería repetirse a las 2-6 semanas, ya que puede volverse positivo y ser útil para reforzar el diagnóstico. El porcentaje de pacientes con Mantoux negativo sería mayor en los VIH positivos, sobre todo en los que tienen un recuento de células CD4+< 200 células/mm3 (Jones, y otros, 1993).

\section{Diagnóstico}

El diagnóstico de certeza de DPTB requiere la demostración de bacilos tuberculosos en el esputo, en el LP o en las muestras de biopsia pleural (BP), o bien la observación, en esta última, de granulomas.

\section{Microbiología}

Una prueba, frecuentemente subestimada, en el diagnóstico de un DP es el cultivo de esputo para micobacterias, cuyas positividades oscilan entre el 41,7 y el 52\%(Valdés, Álvarez, Valle, Pose, \& San José, 1996).

La tinción del LP para el diagnóstico de un DPTB en un paciente inmunocompetente no parece indicada (rentabilidad del 5\%). Sin embargo, en los VIH positivos la tinción puede ser positiva en el $20 \%$ y, por tanto, justificada. El cultivo del LP tiene una mayor rentabilidad, que dependerá del medio de cultivo que se utilice.

Con medios sólidos (Löwenstein-Jensen), el bacilo se identifica en el 12-36,6\% de los casos. Los medios líquidos son más sensibles. Con el sistema BACTEC la rentabilidad del cultivo aumenta del 12 al 24\%, (Ruan, y otros, 2012). Dos aspectos pueden influir en el 


\section{Atención y cuidado en el paciente con tuberculosis por derrame pleural}

Vol. 3, núm. 3., (2019)

Jaqueline Magaly Quisanga Llumiluisa; Nery Hermelinda Balseca Guaman; Marco Antonio Ipiales Miranda; Cristina Mishell Zurita Pozo

resultado: la infección por VIH y el predominio celular del LP. Tanto la tinción como el cultivo del LP son más frecuentemente positivos en los pacientes VIH positivos que en los negativos (37\%vs $0 \%$ para la tinción; $43 \%$ vs $12 \%$ para cultivo en medio sólido; $75 \%$ vs $24 \%$ en medio líquido). Es posible que en estos casos el DPTB sea debido, más que a una reacción a las proteínas tuberculínicas, a un fracaso del sistema inmune que permite a las micobacte-rias permanecer durante más tiempo en el espacio pleural, al ser mínima la actividad micobactericida de los macrófagos. El segundo aspecto que puede influir en el rendimiento del cultivo es el predominio celular. Cuanto mayor es el porcentaje de neutrófilos en el LP, mayor probabilidad de que el cultivo sea positivo, probablemente porque en las fases tempranas de la enfermedad, cuando predominan los neutrófilos, el sistema inmune todavía no es eficaz contra los bacilos. En esta situación, la carga micobacteriana en el LP será mayor. Cuando los macrófagos activados fagocitan el Mycobacterium tuberculosis (MT), la carga micobacteriana intrapleural disminuye, y con ello la probabilidad de que el cultivo sea positivo (Antony, y otros, 2003).

El cultivo MODS (microscopic-observation drug-susceptibility) es un medio líquido, barato, altamente sensible y relativamente rápido que proporciona simultáneamente datos de susceptibilidad a fármacos. Este cultivo fue más rentable que el de Löwenstein-Jensen, tanto en el LP (20\% vs $7 \%$ ) como en la BP ( $81 \%$ vs $51 \%)$, con menor tiempo de espera.

Al ser el DPTB paucibacilar, los métodos microbiológicos utilizados son poco sensibles. Las técnicas de amplificación de las secuencias de ácidos nucleicos específicas de TB permiten detectar el MT del LP, aunque existan pocos bacilos, y en menos tiempo. En un metaanálisis que incluye 38 artículos, la sensibilidad fue del 62\% y la especificidad del 98\%. Es decir, es útil para 


\section{Atención y cuidado en el paciente con tuberculosis por derrame pleural}

Vol. 3, núm. 3., (2019)

Jaqueline Magaly Quisanga Llumiluisa; Nery Hermelinda Balseca Guaman; Marco Antonio Ipiales Miranda; Cristina Mishell Zurita Pozo

confirmar el diagnóstico, pero no para descartarlo. La baja sensibilidad se atribuye a la baja carga bacilar que contienen las muestras, a la probable presencia de sustancias en el LP que inhiban la amplificación, a la secuencia genómica amplificada y al posible secuestro intracelular de las micobacterias. Esta sensibilidad y el precio de cada determinación hacen que no se realice de rutina en el estudio de un DP.

El Xpert es un método de amplificación de ácidos nucleicos automatizado que detecta simultáneamente MT y resistencia a la rifampicina en menos de 2 h45. Su rentabilidad en el diagnóstico de los DPTB es baja (especificidad 100\%, pero la sensibilidad oscila entre el 15 y el 27\%) (Porcel, y otros, 2013).

Biopsia pleural

La BP cerrada ciega es el método más sensible para el diagnóstico de los DPTB. Se pueden observar granulomas en el 50-97\% de los casos (Porcel \& Vives, Etiology and pleural fluid characteristics of large and mas-sive effusions, 2003). Aunque otras enfermedades producen una pleuritis granulomatosa, más del 95\% son debidas a una TB. El cultivo sistemático de la BP contribuye a aumentar la rentabilidad diagnóstica de los DPTB (del 79,8 al 91,5\% con el cultivo). La toracoscopia también puede ser útil, aunque habitualmente no es necesario recurrir a ella. Si el paciente tiene una TB, la toracoscopia puede llegar al 100\% de sensibilidad.

Por tanto, los diagnósticos de certeza tienen limitaciones: baja rentabilidad de cada una de las pruebas, tiempo de espera pro-longado para los resultados de los cultivos, o el uso de técnicas invasoras como la BP. Para mejorar la rentabilidad diagnóstica se ha evaluado la utilidad de la 


\section{Atención y cuidado en el paciente con tuberculosis por derrame pleural}

Vol. 3, núm. 3., (2019)

Jaqueline Magaly Quisanga Llumiluisa; Nery Hermelinda Balseca Guaman; Marco Antonio Ipiales Miranda; Cristina Mishell Zurita Pozo

determinación en el LP de los biomarcado-res que de alguna forma intervienen en la respuesta inmunológica de la TB (fig. 1).

\section{Adenosina desaminasa}

La enzima adenosina desaminasa (ADA), aunque ubicua en su distribución, juega un papel relevante en la proliferación y diferenciación de los linfocitos T. Un metaanálisis de 63 estudios que incluye más de 8.000 DP, 2.796 con DPTB, muestra que la sensibilidad, la especificidad, las razones de probabilidad positiva (RPP) y negativa (RPN) y la odds ratio fueron 92\%, 90\%, 9, 0,10 y 110, respectivamente (Liang, Shi, Wang, Qin, \& Qin, 2008). La ADA tiene 2 isoenzimas: ADA1 y ADA2. La primera se halla en todas las células, mientras que la última solamente se encuentra en los monocitos/macrófagos y se eleva cuando estas células se estimulan por microorganismos vivos en su interior. En los DPTB predomina la ADA2. Las isoenzimas de la ADA se pueden determinar por 3 métodos: a) separación de las fracciones por electroforesis; b) inhibición de la ADA1 mediante eritro-9(2-hidroxi-3-nonil) adenina (EHNA)y c) cálculo de la ADA1 y ADA2 a partir del diferente coeficiente de afinidad de las mismas por los sustratos adenosina y 2'desoxiadenosina. De este se pueden derivar los cocientes 2'desoxiadenosina/adenosina o el ADA1/ADA; el primero incorpora menos errores, al obtenerse los datos a partir de la actividad enzimática y no por cálculos con factores teóricos.

Aunque la rentabilidad de la ADA2 es ligeramente superior (ADA vs ADA2: sensibilidad $100 \%$ para ambas, especificidad $91 \%$ vs $96 \%$; sensibilidad $93,7 \%$ vs $97 \%$, especificidad $89 \%$ vs 94\%), su uso no parece justificado en la práctica clínica. 


\section{Atención y cuidado en el paciente con tuberculosis por derrame pleural}

Vol. 3, núm. 3., (2019)

Jaqueline Magaly Quisanga Llumiluisa; Nery Hermelinda Balseca Guaman; Marco Antonio Ipiales Miranda; Cristina Mishell Zurita Pozo

Los niveles de ADA también se elevan tanto en los DPTB con bajo número de células CD4+65 (sensibilidad 94\%, especi-ficidad 95\%), probablemente porque los monocitos no se venafectados por la infección retroviral, como en aquellos en los que las células predominantes son los neutrófilos.

Una razón podría ser que los neutrófilos liberen citoquinas que atraigan a los monocitos y a los macrófagos, células productoras de ADA-2, al espacio pleural, contribuyendo así a la alta actividad de la ADA en estos derrames. La ADA en LP se determina de forma rutinaria en países donde la prevalencia de TB como causa de DP es alta/moderada. En estos países, en los menores de 35 años la rentabilidad de la ADA es tan elevada que incluso se podría prescindir de la BP para el diagnóstico. Sin embargo, en países donde la prevalencia es baja, el valor predictivo positivo de este test será también bajo, por lo que su utilidad radicaría en poder descartar la enfermedad (alto valor predictivo negativo).

La determinación de la ADA tiene varias limitaciones. Al existir diversos métodos de determinación, los resultados pueden no ser extrapolables, lo que a su vez incidirá en la variabilidad de los puntos de corte utilizados, si bien también pueden influir otros factores, como la etnia de la población. Por tanto, el punto de corte debe-ría establecerse según los resultados de cada centro o, al menos, basándose en los estudios de poblaciones similares y con la misma metodología. La ADA también presenta falsos negativos, pero determinaciones repetida-mente bajas parecen descartar la enfermedad. La ADA no deja de ser un biomarcador inflamatorio, por lo que no sustituye al cultivo ni informa sobre la sensibilidad a los fármacos antituberculosos. Por tanto, en zonas con alto nivel de resistencias micobacterianas, ante un exudado linfocítico 


\section{Atención y cuidado en el paciente con tuberculosis por derrame pleural}

Vol. 3, núm. 3., (2019)

Jaqueline Magaly Quisanga Llumiluisa; Nery Hermelinda Balseca Guaman; Marco Antonio Ipiales Miranda; Cristina Mishell Zurita Pozo

con una ADA elevada sería deseable intentar una BP, enviar muestras para cultivo y testar la sensibilidad a fármacos.

Figura 3. Efecto de la prevalencia del derrame pleural tuberculoso en los valores predictivos positivo y negativo de adenosina desaminasa, para el diagnóstico del mismo (sensibilidad 90\%, especificidad 93\%). Cuando la prevalencia de la enferme-dad es baja, su valor predictivo positivo también lo es, por lo que su utilidad radica en poder descartar la enfermedad (alto valor predictivo negativo). Si la prevalencia es alta, el valor predictivo positivo también está elevado, por lo que puede ser útil para confirmar la enfermedad.

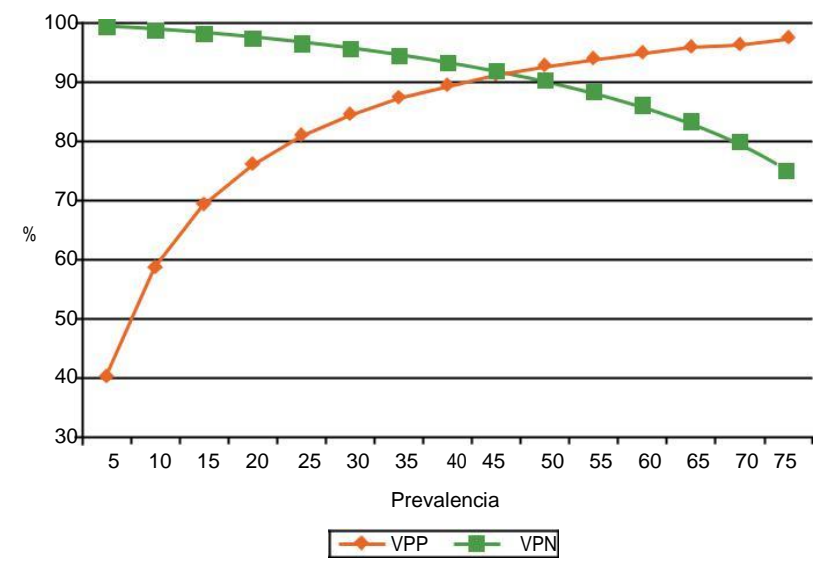

VPN: valor predictivo negativo; VPP: valor predictivo positivo.

Interferón-gamma

El interferón-gamma (IFN- ) es una citoquina liberada por los linfocitos T-CD4+ que aumenta la actividad micobactericida de los macrófagos. Podemos determinar el IFN- presente en el LP o el liberado por las células mononucleares del LP tras su estimulación con antígenos específicos de MT. Al igual que con la ADA, no existe un punto de corte universal, porque los 


\section{Atención y cuidado en el paciente con tuberculosis por derrame pleural}

Vol. 3, núm. 3., (2019)

Jaqueline Magaly Quisanga Llumiluisa; Nery Hermelinda Balseca Guaman; Marco Antonio Ipiales Miranda; Cristina Mishell Zurita Pozo

métodos de determinación no son siempre los mismos. También pueden cursar con valores elevados de IFN- los derrames neoplásicos, especialmente los hematológicos, y los empiemas (Villena, López-Encuentra, Pozo, \& Echave-Sustaeta, 2003). La rentabilidad del IFN- enlos DPTB es ligeramente superior, aunque no significativa, a la de la ADA-

La determinación de IFN- estimulado con antígenos específicos de MT (ESAT-6 y CFP10) podría contribuir al diagnóstico de los DPTB. En un metaanálisis de 7 artículos con 366 pacientes (213 con DPTB), la sensibilidad, la especificidad, la RPP, la RPN y la odds ratio fueron en LP 75\%, 82\%, 3,5, 0,24 y 19, respectivamente, y ligeramente inferiores en sangre. Estos resultados, y su precio, no justifican su determinación en la práctica clínica diaria.

\section{Tabla 1}

Otros biomarcadores utilizados en el diagnóstico de los derrames pleurales tuberculosos

\begin{tabular}{|c|c|c|c|c|c|c|c|c|c|c|}
\hline Biomarcador & Referencia & Ano $^{\sim}$ & DP totales & DPTB & Punto de corte & $\mathrm{S}(\%)$ & $E(\%)$ & VPP (\%) & VPN (\%) & Comentarios \\
\hline Neopterina & 83 & 2005 & 63 & 34 & $30 \mathrm{~mol} / \mathrm{l}$ & 85 & 93 & 94 & 84 & \\
\hline Leptina & 84 & 2006 & 45 & 17 & $9,85 \mathrm{ng} / \mathrm{ml}$ & 82,4 & 82,1 & & & Significativamente peor que la ADA \\
\hline Lisozima LP/S & 85 & 1988 & 138 & 61 & 1,2 & 100 & 87 & 86 & 100 & Utilizada junto a la ADA \\
\hline Lisozima LP/S & 23 & 1993 & 276 & 49 & 1,2 & 67,3 & 90,3 & 60 & 92,7 & Baja sensibilidad \\
\hline Fibronectina & 86 & 1989 & 51 & 14 & - & & & & & $\begin{array}{l}\text { No aporta datos de rentabilidad. } \\
\text { Niveles significativamente mayores } \\
\text { en los DPTB que en los neoplásicos }\end{array}$ \\
\hline RS IL-2 & 87 & 2004 & 55 & 20 & & & & & & $\begin{array}{l}\text { No aporta más datos que el área bajo } \\
\text { la curva: } 0,990 \text { (mejor que la de la } \\
\text { ADA pero peor que la del IFN- ) }\end{array}$ \\
\hline TNF- & 88 & 1999 & 63 & 33 & - & & & & & $\begin{array}{l}\text { No aporta datos de rentabilidad. } \\
\text { Niveles significativamente mayores } \\
\text { en los DPTB que en los neoplásicos }\end{array}$ \\
\hline IL-1 & 88 & 1999 & 63 & 33 & - & & & & & $\begin{array}{l}\text { No aporta datos de rentabilidad. } \\
\text { Niveles significativamente mayores } \\
\text { en los DPTB que en los neoplásicos }\end{array}$ \\
\hline $\begin{array}{l}\text { Activación } \\
\text { complemento } \\
\text { (SC5b-9) }\end{array}$ & 89 & 1992 & 64 & 26 & $2 \mathrm{mg} / \mathrm{l}$ & 100 & 74 & 72 & 100 & Correlación entre ADA y SC5b-9 \\
\hline IL-12 p40 & 90 & 2009 & 96 & 39 & $550 \mathrm{pg} / \mathrm{ml}$ & 92,3 & 70,2 & 67,9 & 93 & Significativamente peor que la ADA \\
\hline IL-6 & 91 & 2003 & 66 & 32 & $4.000 \mathrm{pg} / \mathrm{ml}$ & 90,6 & 79,4 & & & \\
\hline IL-6 & 92 & 2002 & 57 & 9 & - & & & & & $\begin{array}{l}\text { Niveles significativamente mayores } \\
\text { que en los derrames malignos y } \\
\text { paraneumónicos }\end{array}$ \\
\hline CD4+ & 93 & 2005 & 70 & 36 & - & 75,6 & 77,1 & & & $\begin{array}{l}\text { Valores significativamente inferiores } \\
\text { que los de la ADA. Correlación entre } \\
\text { ADA y CD4+ }\end{array}$ \\
\hline IP-10 & 94 & 2009 & 74 & 48 & $28.170 \mathrm{pg} / \mathrm{ml}$ & 80 & 82 & 91 & 64 & \\
\hline & & & & & $4.035 \mathrm{pg} / \mathrm{ml}$ & 100 & 53 & 83 & 100 & \\
\hline \multicolumn{2}{|c|}{ Lipoarabinomanan 94} & 2009 & 74 & 48 & & & & & & No utilidad clínica. No aporta datos \\
\hline
\end{tabular}




\section{Atención y cuidado en el paciente con tuberculosis por derrame pleural}

Vol. 3, núm. 3., (2019)

Jaqueline Magaly Quisanga Llumiluisa; Nery Hermelinda Balseca Guaman; Marco Antonio Ipiales Miranda; Cristina Mishell Zurita Pozo

ADA: adenosina deaminasa; DP: derrame pleural; DPTB: derrame pleural tuberculoso; E: especificidad; IFN- , interferón-gamma; IP-10: proteína inducible 10; LP/S: líquido pleural suero; RS IL-2: receptores solubles interleuquina-2; S: sensibilidad; TNF- : factor- de necrosis tumoral; VPN: valor predictivo negativo; VPP: valor predictivo positivo.

\section{Otros biomarcadores}

La determinación de otros biomarcadores en el LP para el diagnóstico de los DPTB ha tenido una rentabilidad menor que la de la ADA y el IFN- y se resume en la tabla 1.

\section{Celularidad}

Aunque en las 2 primeras semanas el recuento diferencial de células puede revelar un predominio de polimorfonucleares95, lo habitual es que sea de linfocitos (> 50\%). Si este recuento supera el $80 \%$, el diagnóstico diferencial estaría entre TB, linfoma y algunos pocos casos de DP metastásicos, ya que otras posibilidades podrían excluirse por su escasa prevalencia (pleuritis reumatoide y sarcoidosis), por la historia clínica (cirugía de revasculariza-ción mediante by-pass aorto-coronario, rechazo pulmonar agudo y síndrome de uñas amarillas), o por el aspecto del líquido (quilotórax). El recuento de células mesoteliales no suele ser > 5\%99, aunque una cifra superior no excluye el diagnóstico. Más controvertidoes si la presencia de más de un $10 \%$ de eosinófilos en el LP reduce la probabilidad de TB. 


\section{Atención y cuidado en el paciente con tuberculosis por derrame pleural}

Vol. 3, núm. 3., (2019)

Jaqueline Magaly Quisanga Llumiluisa; Nery Hermelinda Balseca Guaman; Marco Antonio Ipiales Miranda; Cristina Mishell Zurita Pozo

\section{Combinación de parámetros}

Varios estudios han desarrollado test diagnósticos basados en la combinación de múltiples variables (tabla 2). Engeneral, todos logran mejorar la rentabilidad de los parámetros utilizados individualmente, pero son poco útiles en la clínica diaria ya que necesitan aplicar complejos cálculos estadísticos que, por otra parte, no dejan de ser arbitrarios. Por el contrario, Bur-gess et al.108, utilizando un valor de ADA > 50 U/l y un cocientelinfocitos/neutrófilos > 0,75, obtienen una elevada rentabilidad diagnóstica.

\section{Tratamiento}

Un DPTB no tratado puede resolverse espontáneamente en 1-4 meses, si bien el 65\% de los casos pueden desarrollar posteriormente una TB pulmonar. De acuerdo con las recomendaciones actuales, un DPTB debe tratarse, como la TB pulmonar, con 4 fármacos (isoniazida, rifampicina, piracinamida y etambutol)durante 2 meses e isoniazida y rifampicina durante 4 meses más (2HRZE/4HR)(Anibarro, Vidal, Esteban, \& Blanquer, 2010). El etambutol se añade por si la resistencia primaria a isoniazida es elevada (> 4\%). Si esto no sucede y no hay afectación pulmonar, al ser el LP paucibacilar, una pauta sin etambutol sería igualmente útil.

\section{Tabla 2}

Combinación de variables para el diagnóstico de los derrames pleurales tuberculosos

\begin{tabular}{|c|c|c|c|c|}
\hline Referencia & Ano $^{\sim}$ & Combinación utilizada & Características & Rentabilidad \\
\hline 81 & 2009 & Edad, proteínas & DPTB vs no-DPTB & Sensibilidad $54 \%$, especificidad $89 \%$ \\
\hline 101 & 2003 & ADA, edad, temperatura, recuento de hematíes & DPTB vs DP neoplásicos & Sensibilidad $95 \%$, especificidad $94 \%$ \\
\hline 102 & 2001 & $\begin{array}{l}\text { Edad, recuento de leucocitos, prueba de la tuberculina, } \\
\text { exudado sanguinolento }\end{array}$ & DPTB vs no-DPPTB & Sensibilidad $90 \%$, especificidad $87 \%$ \\
\hline 103 & 2009 & $\begin{array}{l}\text { ADA, globulinas, ausencia de células malignas en el líquido } \\
\text { pleural }\end{array}$ & DPTB vs DP neoplásicos & Sensibilidad $94,5 \%$, especificidad $99,5 \%$ \\
\hline 104 & 2000 & ADA, IFN- , NAAT & DPTB vs no-DPTB & Sensibilidad $90,5 \%$, especificidad $88,2 \%$ \\
\hline 105 & 2007 & ADA, proteínas, LDH, recuento leucocitos, linfocitos (\%) & DPTB vs no-DPTB & Sensibilidad y especificidad $>95 \%$ \\
\hline 106 & 1996 & ADA, cociente linfocitos/neutrófilos & DPTB vs no-DPTB & Sensibilidad $88 \%$, especificidad $95 \%$ \\
\hline
\end{tabular}




\section{Atención y cuidado en el paciente con tuberculosis por derrame pleural}

Vol. 3, núm. 3., (2019)

Jaqueline Magaly Quisanga Llumiluisa; Nery Hermelinda Balseca Guaman; Marco Antonio Ipiales Miranda; Cristina Mishell Zurita Pozo

ADA: adenosina deaminasa; DPTB: derrame pleural tuberculoso; IFN- , interferón-gamma;

LDH, lactato deshidrogenasa; NAAT: test de amplificación de ácidos nucleicos.

El tratamiento en la coinfección TB-VIH es el estándar (2HRZE/4HR). Existe una mayor prevalencia de resistencias a fármacos antituberculosos, que se tratan como en los pacientes inmunocompetentes, y puede surgir una interacción de la rifam-picina con los inhibidores de la proteasa y algún inhibidor de la transcriptasa inversa no análogo de nucleósido. En estos casos existirían varias opciones, como completar el tratamiento antitu-berculoso antes de comenzar con los antirretrovirales o introducir estos a los 2 meses, o a las 2-8 semanas de haber iniciado el men-cionado tratamiento, dependiendo del recuento de células CD4+. Si no se puede utilizar la rifampicina, el tratamiento es menos eficaz y puede utilizarse la rifabutina o prolongar la fase de mantenimiento con isoniazida y etambutol hasta los 18 meses.

Algunos pacientes $(16 \% ; 10 / 61)$ pueden desarrollar un aumento paradójico del DP durante el tratamiento. Esto se atribuye a un «rebote inmunológico» al coincidir, al iniciar el tratamiento, una excesiva carga antigénica en el LP, como resultado de una rápida lisis micobacteriana, con una mejoría de la inmunidad celular, lo que produciría una nueva reacción de hipersensibilidad.

Existe cierta controversia sobre si los corticoides orales podrían, a través de su acción antiinflamatoria, acelerar la reabsorción del líquido y prevenir las adherencias pleurales. En 3 ensayos clínicos no se observaron diferencias en el desarrollo de engrosamientos pleurales residuales, adherencias o deterioro de la función pulmonar, si bien, en uno de ellos, en los tratados con corticoides los días con fiebre fueron menores y la resolución del DP se acortó. En 


\section{Atención y cuidado en el paciente con tuberculosis por derrame pleural}

Vol. 3, núm. 3., (2019)

Jaqueline Magaly Quisanga Llumiluisa; Nery Hermelinda Balseca Guaman; Marco Antonio Ipiales Miranda; Cristina Mishell Zurita Pozo

una serie con DPTB-VIH la administración de corticoides se asoció a un mayor riesgo de sarcoma de Kaposi.

El drenaje rutinario del DP no parece recomendado. Sin embargo, si existe disnea por un gran DP, sí podría llevarse acabo119. Si el DPTB está loculado, el uso de fibrinolíticos aceleraría la resolución del DP y reduciría la incidencia de engrosamiento pleural residual. Este último podría relacionarse con la magnitud del cambio inicial de los niveles de $\mathrm{pH}$, glucosa y del factorde necrosis tumoral (TNF).

\section{Conclusiones.}

La incidencia de TB, y por tanto de DPTB, está disminuyendo. Afecta fundamentalmente a pacientes jóvenes, mayoritariamente entre 15 y 44 años, y casi las dos terceras partes son hombres. La presentación clínica varía dependiendo de la población afectada. Los diagnósticos de certeza presentan limitaciones, por lo que se ha utilizado la determinación en el LP de diversos biomarcadores. La asociación de la ADA y el porcentaje de linfocitos pueden ser útiles para el diagnóstico. La prevalencia de DPTB influye sobre los valores predictivos positivo y negativo de la ADA y el tratamiento es el de cualquier TB. No parece recomendable añadir corticoides y el drenaje torácico podría contribuir, en los grandes derrames, a una mejoría más rápida de los síntomas.

\section{Bibliografía.}

Anibarro, L., Vidal, R., Esteban, J., \& Blanquer, R. (2010). Consensus document on the diagnosis, treatment and prevention of tuberculosis. Arch Bronconeumol., 255-74. 


\section{Atención y cuidado en el paciente con tuberculosis por derrame pleural}

Vol. 3, núm. 3., (2019)

Jaqueline Magaly Quisanga Llumiluisa; Nery Hermelinda Balseca Guaman; Marco Antonio Ipiales Miranda; Cristina Mishell Zurita Pozo

Antony, V., Repine, J., Harada, R., Good, J., Sahn, S. 1., \& 27:. (2003). Inflammatory responses in tuberculous pleuritis. . Acta Cytol., 355-61.

Awil, P., Bowlin, S., \& Daniel, T. (1997). Radiology of pulmonary tuberculosis and human immunodeficiency virus infection in Gulu, Uganda. Eur Respir J, 615-8.

Baumann, M., Nolan, R., Petrini, M., Lee, Y., Light, R., \& Schneider, E. (2007). Pleural tuberculosis in the United States. Incidence and drug resistance. . Chest., 1125-32.

Bolívar, J. (2015). Investigación Documental. México. Pax.

Castro, J. (2016). Técnicas Documentales. México. Limusa.

Davila, A. (2015). Concepto de terminos cientificos. Caracas: Oasis.

DetallePublicacion.aspx. (2009). Evolución en el periodo 1996-2009 (publicación electrónica). Obtenido de nforme de tuberculosis en Galicia: características de los casos de tuberculosis de Galicia en el ano 2009.: http://www.sergas.es/Publicaciones/

Jones, B., Young, S., Antoniskis, D., Davidson, P., Kramer, F., \& Barnes, P. (1993). Relationship of the manifestations of tuberculosis to CD4 cell count in patients with human immunodeficiency virus infection. Am Rev Respir Dis, 1292-7.

Leibowitz, S., Kennedy, L., \& Lessof, M. (1973). The tuberculin reaction in the pleural cavity and its suppression by antilymphocyte serum. Bj J Exp Pathol, 152-62.

Liang, Q., Shi, H., Wang, K., Qin, S., \& Qin, X. (2008). Diagnostic accuracy of ade-nosine deaminase in tuberculous pleurisy: A meta-analysis. Respir Med, 744-54.

Light, R. (2010). Update on tuberculous pleural effusion. Respirology, 451-458.

Pereyra, M., Ferreiro, L., \& Valdés, L. (2013). Pulmón no expansible. Arch Bronconeumol, 639.

Porcel, J., \& Vives, M. (2003). Etiology and pleural fluid characteristics of large and mas-sive effusions. Chest, 978-83.

Porcel, J., Palma, R., Valdés, L., Bielsa, S., San José, E., \& Esquerda, A. (2013). GeneXpert MTB/RIF in pleural fluid for diagnosing tuberculosis. Int J Tuberc Lung Dis., 1217-9.

Ruan, S., Chuang, Y., Wang, J., Lin, J., Chien, J., \& Huang, C. (2012). Revisiting tuberculous pleurisy: Pleural fluid characteristics and diagnostic yield of myco-bacterial culture in an endemic area. Thorax., 822-7.

Valdés, L., Álvarez, D., Valle, J., Pose, A., \& San José, E. (1996). La etiología de los derrames pleurales en un área con alta incidencia de tuberculosis. Chest, 158-62. 


\section{Atención y cuidado en el paciente con tuberculosis por derrame pleural}

Vol. 3, núm. 3., (2019)

Jaqueline Magaly Quisanga Llumiluisa; Nery Hermelinda Balseca Guaman; Marco Antonio Ipiales Miranda; Cristina Mishell Zurita Pozo

Villena, V., López-Encuentra, A., Pozo, F., \& Echave-Sustaeta, J. (2003). Interferon gamma levels in pleural fluid for diagnosis of tuberculosis. Am J Med, 365-70.

World Health Organization. (2012). WHO Report Global Tuberculosis Report. WHO/HTM/TB/2012.6. 